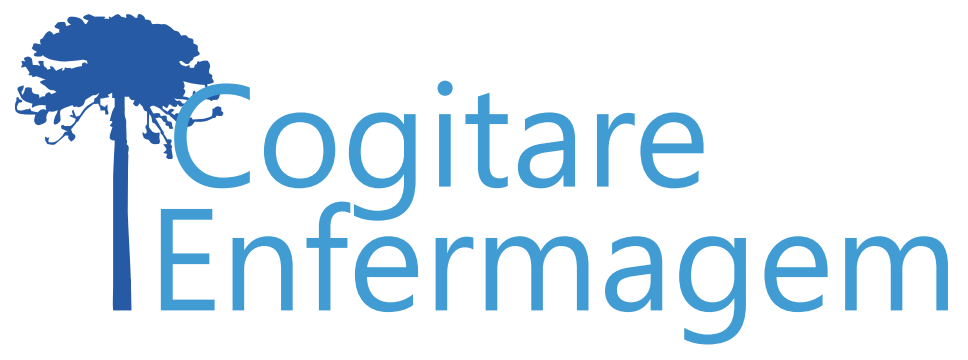

\title{
COGNIÇÃO E FRAGILIDADE DE IDOSOS DA COMUNIDADE
}

Renata Rabelo Pereira1, Cleane Rosa Ribeiro da Silva², Selene Cordeiro Vasconcelos ${ }^{3}$, Lucineide Alves Vieira Braga ${ }^{4}$, Edilene Araújo Monteiro ${ }^{5}$, Maria de Lourdes de Farias Pontes 6

\section{RESUMO}

Objetivo: analisar a relação entre o desempenho cognitivo e a fragilidade de idosos da comunidade. Método: estudo transversal, observacional e quantitativo, realizado no período de agosto a dezembro de 2016, com 126 idosos cadastrados em uma Unidade de Saúde da Família de João Pessoa-PB. O Mini Exame do Estado Mental foi utilizado para a avalição cognitiva e a Escala de Fragilidade de Edmonton para a fragilidade. Foi realizada análise descritiva e inferencial, aplicaramse testes de Qui-Quadrado de Independência, Spearman, Kendall, Coeficiente de Cramér e Razão de Chance.

Resultados: na avaliação do desempenho cognitivo, verificou-se que 24 (19\%) idosos manifestaram déficit cognitivo e $50(39,6 \%)$ demonstraram ser frágeis. Osidosos com déficit cognitivo apresentaram 2,56 (IC95\%: 0,55-0,96) mais chance de serem frágeis.

Conclusão: evidenciou-se relação estatí́stica significante entre o desempenho cognitivo com a fragilidade em idosos da comunidade, evidenciando que idosos que têm déficit cognitivo apresentam maior probabilidade de serem frágeis.

DESCRITORES: Enfermagem geriátrica; Cognição; Idoso; Idoso fragilizado.

\section{COMO REFERENCIAR ESTE ARTIGO:}

Pereira RR, CRR da, Vasconcelos SC, Braga LAV, Monteiro EA, Pontes M de L de F. Cognição e fragilidade de idosos da comunidade. Cogitare enferm. [Internet]. 2019 [acesso em "colocar data de acesso, dia, mês abreviado e ano"]; 24. Disponível em: http://dx.doi.org/10.5380/ce.v24i0.60578.

\section{(c) (1)}

Este obra está licenciado com uma Licença Creative Commons Atribuição 4.0 Internacional.

${ }^{1}$ Enfermeira. Mestre em Enfermagem. Universidade Federal da Paraíba. João Pessoa, PB, Brasil. () ${ }^{2}$ Enfermeira. Mestre em Enfermagem. Universidade Federal da Paraíba. João Pessoa, PB, Brasil.

${ }^{3}$ Enfermeira. Doutora em Neuropsiquiatria e Ciências do Comportamento. Docente de Enfermagem da Universidade Federal da Paraíba. João Pessoa, PB, Brasil. (

${ }^{4}$ Enfermeira. Doutoranda em Enfermagem. Universidade Federal da Paraíba. João Pessoa, PB, Brasil. $\bigcirc$

${ }^{5}$ Enfermeira. Doutora em Enfermagem. Docente de Enfermagem da Universidade Federal da Paraíba. João Pessoa, PB, Brasil. ()

${ }^{6}$ Enfermeira. Doutora em Enfermagem. Docente de Enfermagem da Universidade Federal da Paraíba. João Pessoa, PB, Brasil. () 


\title{
COGNITION AND FRAILTY IN COMMUNITY-DWELLING ELDERLY
}

\begin{abstract}
Objective: To analyze the relationship between cognitive performance and frailty in communitydwelling elderly.

Method: Cross-sectional, observational and quantitative study conducted in August-December 2016, with 126 elderly registered in a Family Health Unit of João Pessoa, state of Paraíba. Mini Mental State Examination (MMSE) was used for cognitive assessment and the Edmonton Frailty Scale was used for the assessment for frailty. Descriptive and inferential analysis were performed, and Pearson's chi-square test of independence, Nonparametric Spearman and Kendall tests and Cramér's V Coefficient and Odds Ratio (OR) tests were applied.

Results: The cognitive performance assessment revealed that 24 (19\%) elderly had cognitive impairment and 50 (39.6\%) were frail. Elderly people with cognitive impairment were 2.56 (95\% Cl: 0.55-0.96) more likely to be frail.

Conclusion: There was a statistically significant relationship between cognitive performance and frailty among community-dwelling elderly, demonstrating that older people with cognitive impairment are more likely to be frail.
\end{abstract}

DESCRIPTORS: Geriatric nursing; Cognition; Elderly; Frail elderly.

\section{COGNICIÓN Y FRAGILIDAD DE ANCIANOS DE LA COMUNIDAD}

\begin{abstract}
RESUMEN
Objetivo: analizar la relación entre el desempeño cognitivo y la fragilidad de ancianos de la comunidad.

Método: estudio trasversal, observacional y cuantitativo, que se realizó en el periodo de agosto a diciembre de 2016, con 126 ancianos registrados en una Unidad de Salud de Familia de João Pessoa, PB. Se utilizó el Mini Examen de Estado Mental para la evaluación cognitiva y la Escala de Fragilidad de Edmonton para la fragilidad. Se realizó análisis descriptivo y de inferencia, además de la aplicación de pruebas de Chi Cuadrado de Independencia, Spearman, Kendall, Coeficiente de Cramér y Razón de Chance.

Resultados: en la evaluación del desempeño cognitivo, se verificó que 24 (19\%) ancianos manifestaron déficit cognitivo y $50(39,6 \%)$ demostraron fragilidad. Los ancianos con déficit cognitivo presentaron 2,56 (IC 95\%:0,55-0,96) más probabilidad de fragilidad.

Conclusión: se evidenció relación estadística significante entre el desempeño cognitivo y la fragilidad en ancianos de la comunidad, apuntando que ancianos que tienen déficit cognitivo presentan más probabilidad de ser frágiles.
\end{abstract}

DESCRIPTORES: Enfermería geriátrica; Cognición; Anciano; Anciano fragilizado. 
O envelhecimento da população é uma temática discutida mundialmente por pesquisadores de diversas áreas com a finalidade de formular estratégias que contribuam com o envelhecimento saudável e ativo.

Nos países em desenvolvimento, o aumento populacional entre as pessoas idosas caracteriza-se como uma realidade, tornando necessário o desenvolvimento de políticas específicas para essa população, uma vez que novas demandas de cuidado surgem com as mudanças dos padrões de morbimortalidade ${ }^{(1)}$. A fragilidade em idosos emerge na atualidade como um destes desafios ${ }^{(2)}$.

O termo fragilidade destaca-se nas últimas dé $c$ cadas em estudos sobre o envelhecimento, e, apesar de ser amplamente utilizado, não há um consenso sobre a sua definição. Assim, a fragilidade caracteriza-se como um conceito em evolução que apresenta uma pluralidade de definições operacionais, fundamentadas em distintos modelos teóricos, o que aumenta a complexidade na compreensão do processo de fragilizaçãa ${ }^{(2)}$. Dentre os referenciais teóricos, o grupo Canadian Iniciative on Frailty and Aging (CIF-A) baseou o construto da fragilidade em uma abordagem holística e multidimensional, determinada por fatores biológicos, psicológicos e sociais relacionados ao curso de vida da pessoa idosa(3).

Indivíduos frágeis compõem um subconjunto de idosos portadores de maior suscetibilidade a desfechos desfavoráveis a saúde, tais como morte, incapacidade e hospitalização, em virtude da redução de sua capacidade para responder às condições adversas, o que os predispõem a doenças crônicas, anorexia, sarcopenia, osteopenia, dependências e déficits cognitivos ${ }^{(4)}$.

Os distúrbios cognitivos frequentemente associam-se à fragilidade em idosos ${ }^{(5-7)}$. Estes geralmente apresentam queixa de esquecimento e outras alterações cognitivas com frequência. Estudos mostram que o envelhecimento, além de repercutir na memória, pode se relacionar também ao declínio das funções executivas e da linguagem ${ }^{(5-7)}$. Alterações nas funções cognitivas, juntamente com variáveis sociais, econômicas, demográficas e de saúde, podem ser fatores de risco para fragilidade ${ }^{(5)}$.

Nos países em desenvolvimento da América Latina, dentre eles o Brasil, o processo de envelhecimento ocasiona impacto negativo na saúde, econômico e social, com predomínio de fragilidade de $7,7 \%$ a $42,6 \%{ }^{(8)}$. Esta variação ocorre em decorrência dos critérios adotados para rastreio da síndrome e das características sociodemográficas e culturais, - que pode contribuir para as variações nos percentuais da fragilidade ${ }^{(9)}$. O processo de fragilização desses indivíduos necessita de investigação aprofundada, no sentido de identificar os fatores passíveis de prevenção, bem como os subsídios para elaboração de intervenções ${ }^{(3-4,9)}$.

Nesse sentido, a síndrome da fragilidade tem sido foco de estudos na área da gerontologia, tendo em vista as repercussões sobre os indivíduos idosos, as suas famílias e a sociedade. Apesar de recentes iniciativas, existe escassez de estudos nacionais que avaliem a relação entre o desempenho cognitivo com a fragilidade da pessoa idosa. Assim, frente ao acelerado e complexo crescimento da população idosa, e por tratar-se de um grupo que apresenta maior probabilidade de surgimento de fragilidade, distúrbios cognitivos, com múltiplas condições crônicas ou incapacitantes, o presente estudo tem como objetivo investigar a relação entre o desempenho cognitivo e a fragilidade de idosos da comunidade.

\section{MÉTODO}

Trata-se de estudo transversal, observacional e quantitativo, realizado em uma 
Unidade de Saúde da Família, no município de João Pessoa-PB, Brasil.

Definiram-se como seguintes critérios para participação no estudo: ter idade igual ou superior de 60 anos, de ambos os sexos, ser cadastrado na Unidade de Saúde da Família escolhida e capaz de responder aos instrumentos de coleta de dados. Foram excluídos do estudo os idosos que apresentassem alguma demência já diagnosticada, alterações na comunicação e audição ou acamados pela impossibilidade de aplicação da Escala de Fragilidade de Edmonton.

A população do estudo correspondeu a 186 indivíduos. O tamanho da amostra foi definido utilizando o cálculo para populações finitas com intervalo de confiança de $95 \%$ $\left(a=0,05\right.$, que fornece $\left.Z_{0,05 / 2}=1,96\right)$, prevalência estimada de $50 \%(p=0,50)$ e margem de erro de $5 \%($ Erro=0,05), o que correspondeu a 126 participantes.

A coleta de dados foi realizada entre os meses de agosto a dezembro de 2016, por meio por meio de entrevistas nas residências dos sujeitos, com duração aproximada de 40 minutos e realizadas por pesquisadores treinados para obtenção das informações, acompanhados por um Agente Comunitário de Saúde. Utilizou-se um instrumento semiestruturado para obtenção de dados referentes ao perfil sociodemográfico, o Mini Exame do Estado Mental (MÉEM) e a Escala de Fragilidade de Edmonton.

O MEEM, traduzido e validado para a população brasileira ${ }^{(10)}$, foi utilizado para a avaliação da função cognitiva dos idosos. É um teste semi-estruturado composto por questỗes agrupadas em sete categorias e pontuadas com o objetivo de avaliar funções específicas: orientação temporal (05 pontos), orientação espacial (05 pontos), memória imediata (03 pontos), cálculo e atenção (05 pontos), evocação das palavras (03 pontos), linguagem (08 pontos) e construção visual (01 ponto). O escore total pode variar de zero a 30 pontos, sendo utilizados pontos de corte de acordo com a escolaridade do respondente: 13 pontos para analfabetos, 18 para baixa (de 1 a 4 anos incompletos) e média escolaridade (de 4 a 8 anos incompletos) e 26 para alta escolaridade (> 8 anos) $)^{(10)}$.

A Escala de Fragilidade de Edmonton (EFS) foi utilizada para mensurar o grau de fragilidade dos idosos. Elaborada na Universidade de Alberta, Edmonton, Canadá, traduzida e validada no Brasil em $2008^{(11)}$. Tem como objetivo avaliar nove domínios: cognição, estado geral de saúde, independência funcional, suporte social, uso de medicamentos, nutrição, humor, continência e desempenho funcional. $O$ escore total varia de 0 a 17 pontos, representando o nível mais elevado de fragilidade. Categoriza-se o idoso, segundo a pontuação alcançada: 0-4, não apresenta fragilidade; 5-6, aparentemente vulnerável; 7-8, fragilidade leve; 9-10, fragilidade moderada; 11 ou mais, fragilidade severa ${ }^{(6)}$. Essa variável também pode ser dicotomizada em frágil (fragilidade leve, moderada e severa) e não frágil (não apresenta fragilidade e aparentemente vulnerável), e considerou-se o ponto de corte de sete pontos para classificar o indivíduo como portador de fragilidade ${ }^{(11)}$.

Os dados foram digitados e organizados no programa Excel, posteriormente analisados no Software $R$ através de estatística descritiva e inferencial. Foi utilizado o teste de Kolmogorov-Smirnov para a verificação da normalidade das variáveis. Para associação entre as variáveis dicotômicas, aplicou-se o teste Qui-quadrado de independência de Pearson. Na análise da relação entre as variáveis, foram utilizados os testes não paramétricos de Spearman, Kendall e o coeficiente de Cramér. Também foi realizado a Razão de Chance e o Intervalo de Confiança de $95 \%$. O nível de significância adotado no estudo foi de $10 \%$.

O estudo atendeu às normas da Resolução 466/12(12) que envolve seres humanos e foi aprovado pelo Comitê de Ética do Centro de Ciências da Saúde da Universidade Federal da Paraíba, sob o número de parecer 1.702.542. 
referiram faixa etária entre 60 e 69 anos e 53 (42,1\%) estado civil casado. Em relação ao arranjo domiciliar $40(31,6 \%)$ idosos moravam com o cônjuge. Quanto à escolaridade, 63 (50\%) declararam ser analfabetos e $60(47,6 \%)$ possuíam renda familiar de 1 a 3 salários mínimos.

Quanto à avaliação do desempenho cognitivo, a maioria dos participantes não apresentaram comprometimento 102 (81\%) e 24 (19\%) manifestaram déficit cognitivo (Tabela 1).

Tabela 1 - Desempenho cognitivo de idosos da comunidade. João Pessoa, PB, Brasil, 2016

\begin{tabular}{lcc} 
Desempenho cognitivo & $\mathbf{n}$ & $\%$ \\
\hline Sem déficit cognitivo & 102 & 81 \\
\hline Com déficit cognitivo & 24 & 19 \\
\hline Total & 126 & 100
\end{tabular}

$\mathrm{Na}$ avaliação da fragilidade, os resultados demonstraram que o maior percentual de idosos não são frágeis $76(60,4 \%)$, sendo que 39 (31\%) não apresentaram fragilidade e 37 $(29,4 \%)$ são aparentemente vulneráveis. Dentre os que se apresentaram frágeis, $50(39,6 \%)$ houve predomínio de fragilidade leve $32(25,4 \%)$ (Tabela 2$)$.

Tabela 2 - Distribuição da classificação de fragilidade de idosos da comunidade. João Pessoa, PB, Brasil, 2016

\begin{tabular}{lcc} 
Classificação de fragilidade & $\mathbf{n}$ & $\%$ \\
\hline Não frágil & 76 & 60,4 \\
\hline Não apresentaram fragilidade & 39 & 31 \\
\hline Aparentemente vulnerável & 37 & 29,4 \\
\hline Frágil & 50 & 39,6 \\
\hline Fragilidade leve & 32 & 25,4 \\
\hline Fragilidade moderada & 16 & 12,7 \\
\hline Fragilidade grave & 2 & 1,5 \\
\hline Total & 126 & 100
\end{tabular}

Ao relacionar o estado cognitivo com a fragilidade, identificou-se significância estatística entre as variáveis, tanto na classificação do grau de fragilidade em 5 categorias (Tabela 3) como de forma dicotomizada (Tabela 4). Os testes de Spearman e Kendall apresentaram P-valores similares $(0,023)$ e medidas de associação positivas $(\rho=0,203$ e $\tau=0,186$ ) (Tabela 3), e o Qui-quadrado de Pearson apresentou valor-p de 0,065, Coeficiente de Contingência $C$ positivo, demonstrando uma relação não tão forte, porém apresentou 
uma razão de chance expressiva de 2,56 (Tabela 4). Estes resultados evidenciam que idosos que têm déficit cognitivo aumenta em mais que o dobro a chance de ser um idoso fragilizado.

Tabela 3 - Associação entre o grau de fragilidade e estado cognitivo de idosos da comunidade. João Pessoa, PB, Brasil, 2016

Estado

Grau de Fragilidade

\begin{tabular}{cccccc}
\hline $\begin{array}{c}\text { Sem } \\
\text { Fragilidade }\end{array}$ & $\begin{array}{c}\text { Aparentemente } \\
\text { Vulnerável }\end{array}$ & $\begin{array}{c}\text { Fragilidade } \\
\text { Leve }\end{array}$ & $\begin{array}{c}\text { Fragilidade } \\
\text { Moderada }\end{array}$ & $\begin{array}{c}\text { Fragilidade } \\
\text { Grave }\end{array}$ & $\begin{array}{c}\text { Valor p e } \\
\text { Medida de } \\
\text { Associação }\end{array}$ \\
\hline
\end{tabular}

\begin{tabular}{lccccccccccc}
\hline Cognitivo & $\mathrm{n}$ & $\%$ & $\mathrm{n}$ & $\%$ & $\mathrm{n}$ & $\%$ & $\mathrm{n}$ & $\%$ & $\mathrm{n}$ & $\%$ & \\
\hline $\begin{array}{l}\text { Possui déficit } \\
\text { cognitivo }\end{array}$ & 4 & 10,3 & 6 & 16,2 & 8 & 25 & 5 & 31,3 & 1 & 50 & $\begin{array}{c}0,023^{*} \\
\rho=0,203^{*}\end{array}$ \\
\hline $\begin{array}{l}\text { Não possui } \\
\text { déficit cognitivo }\end{array}$ & 35 & 89,7 & 31 & 83,8 & 24 & 75 & 11 & 68,7 & 1 & 50 & $\begin{array}{c}0,023^{\star *} \\
\mathrm{~T}=0,186^{* *}\end{array}$
\end{tabular}

*Teste de Spearman **Teste de Kendall

Tabela 4 -Associação entre o grau de fragilidade dicotomizado e estado cognitivo de idosos da comunidade. João Pessoa, PB, Brasil, 2016

\section{Desempenho cognitivo}

Grau de Fragilidade dicotomizado

P-valor

Não apresentou fragilidade
ou é aparentemente
vulnerável (escore $<7$ )

n $\%$
Fragilidade leve, moderada ou grave (escore $\geq 7$ )
$C$ de Cramér

n

$\%$

n

$\%$

Razão de

Chance

(IC95\%)

\begin{tabular}{llllll}
\hline Possui déficit cognitivo & 10 & 13,2 & 14 & 28 & $\begin{array}{c}0,065^{*} \\
\mathrm{C}=0,164\end{array}$ \\
\hline $\begin{array}{l}\text { Não possui déficit } \\
\text { cognitivo }\end{array}$ & 66 & 86,8 & 36 & 72 & $\begin{array}{c}\mathrm{RC}=2,56 \\
(0,55-0,96)\end{array}$
\end{tabular}

*Teste Qui-Quadrado de Independência C: Coeficiente de contingência RC: Razão de Chance

\section{DISCUSSÃO}

Dentre os idosos que fizeram parte do estudo, a maioria era do sexo feminino. Resultado semelhante foi encontrado em um estudo epidemiológico de base populacional realizado com 2055 idosos $^{(13)}$ e em pesquisa sobre rastreamento de problemas de saúde em idosos na Atenção Básica ${ }^{(14)}$. Esse padrão reflete o processo da feminização da velhice, que se configura por meio do predomínio das mulheres na população idosa(13-14), realidade que tem sido largamente analisada e discutida.

As mulheres constituem a maioria da população idosa em todas as regiões do mundo, e as estimativas são de que as mulheres vivam, em média, de cinco a sete anos a mais que 
os homens. De acordo com dados epidemiológicos do Brasil, o contingente feminino, dentre a população idosa, passou de 2,2\%, em 1940 para 4,7\% em 2000 e 6\% em 2010 (15)'.

A faixa etária predominante na amostra foi de idosos jovens. Segundo dados do Instituto Brasileiro de Geografia e Estatística (IBGE), a maior proporção de idosos na popula-ção brasileira tem entre 60-69 anos, mas a proporção de idosos com 70 anos ou mais tem aumentado(16).

A maioria dos idosos participantes da pesquisa era casada. Estudo com idosos na Atenção Básica revelaram dado aproximado(17). No quesito arranjo domiciliar, predominou idosos que referiram morar com seus cônjuges, situação que difere das encontradas em outros estudos ${ }^{(17-18)}$.

Ao analisar a escolaridade, verificou-se maior frequência de idosos não alfabetizados. Infere-se que tal achado esteja relacionado às dificuldades de acesso à escola, comum no passado, além de fatores culturais em que não se valorizava a educação escolar, priorizando o trabalho em detrimento da educação(19). No que diz respeito à renda, recebiam de 1 a 3 salários mínimos. Em outros estudos têm sido identificados a baixa condição financeira e o baixo nível de escolaridade entre as pessoas idosas ${ }^{(17,20)}$.

Os idosos com maior nível de escolaridade e renda, geralmente, demonstram maior adesão às práticas de autocuidado, incluindo o uso correto de medicamentos, de meios de transporte e de comunicação, enquanto os de menor poder aquisitivo e intelectual estão mais suscetíveis às doenças $e$, consequentemente, necessitam de maior atenção à saúde ${ }^{(18,21)}$.

Identificou-se que a maioria dos idosos não apresentou déficit cognitivo. Este dado corrobora com estudos realizados em idosos residentes no domicílio(2,5,22). $\mathrm{Na}$ avaliação da fragilidade, verificou-se maior percentual de idosos não frágeis. Na adaptação e validação da Escala de Fragilidade de Edmonton para o Brasil, a frequência de idosos não frágeis foi de $68,6 \%{ }^{(11)}$. Uma pesquisa conduzida com idosos residentes em Ribeirão Preto-SP identificou que $62,1 \%$ dos participantes não apresentaram fragilidade ${ }^{(23)}$.

O elevado percentual de não frágeis e sem déficit cognitivo pode ser justificado pela predominância de idosos jovens na amostra estudada. A produção científica aponta a idade como um preditor da fragilidade e de comprometimento cognitivo, demonstrando que os longevos são mais vulnerável a esses agravos ${ }^{(5,9,23-24)}$. Entretanto, ambas as situações não devem ser entendidas como sinônimo de velhice.

Ao relacionar a fragilidade com a presença ou não de déficit cognitivo, os resultados demonstraram associação significativa entre essas variáveis, evidenciando que idosos que têm déficit cognitivo apresentam maior probabilidade de serem frágeis. Estes dados corroboram com outras pesquisas que apontam que as alterações cognitivas podem estar diretamente relacionadas à fragilidade $e^{(1,24-25)}$.

Estudo de base populacional realizado com idosos que vivem no domicílio em dois municípios brasileiros, João Pessoa-PB e Ribeirão Preto-SP, evidenciou que a diminuição do desempenho cognitivo se correlacionou ao aumento da fragilidade em ambas as cidades $^{(25)}$. Uma pesquisa longitudinal realizada com 1.045 idosos na China identificou 2,28 (IC95\%: 1,02-5,08) vezes mais chances dos idosos frágeis possuírem comprometimento cognitivo ${ }^{(26)}$.

$\mathrm{Na}$ longevidade, os danos cognitivos e físicos são frequentes e podem ser intensificados pelo acúmulo das repercussões inerentes ao processo de envelhecimento e/ ou em virtude da presença de morbidades crônicas ${ }^{(27)}$. Idosos com o desempenho cognitivo comprometido geralmente têm mais dificuldade para se alimentar, exercitar, caminhar, o que pode levar à perda de peso e prejuízo das funções motoras, favorecendo o início e progressão da síndrome da fragilidade ${ }^{(28)}$.

Um estudo de coorte prospectivo desenvolvido com 1.751 idosos residentes em 
comunidade, por um período de cinco anos, evidenciou taxa de risco para mortalidade de 2,17 (IC95\%: 1,69-2,80) nos idosos com déficit cognitivo, de 2,02 (IC95\%:1,53-2,68) entre os frágeis, e para os idosos que apresentaram ambas condições o risco aumentou para 3,57 (IC95\%: 2,75-4,62)(29). Demonstrando que a fragilidade e o comprometimento cognitivo foram preditores para a taxa de mortalidade em idosos, sendo a coexistência desses agravos mais prejudicial(29).

Diante disso, no âmbito da enfermagem, torna-se fundamental inserir a avaliação e o monitoramento do desempenho cognitivo e da fragilidade no processo de cuidar da pessoa idosa, o que favorece a identificação de grupos de risco e fornece subsídios para o planejamento de plano de cuidado, com ações e intervenções de prevenção e promoção eficazes, a fim de evitar e/ou reduzir agravos, oportunizando melhorias na condição de vida desse segmento populacional ${ }^{(4,25-26)}$.

O presente estudo apresentou como limitação o delineamento transversal por não possibilitar a relação de causa e efeito entre as variáveis. Sugere-se a realização de pesquisas longitudinais com o intuito de investigar o impacto do déficit cognição, da fragilidade e sua coexistência na vida do idoso em longo prazo.

\section{CONCLUSÃO}

Os resultados deste estudo revelaram relação estatística significante entre o desempenho cognitivo com a fragilidade de idosos da comunidade. O déficit cognitivo foi identificado como um fator de risco para a síndrome da fragilidade.

Nessa perspectiva, torna-se imprescindível a avaliação periódica do desempenho cognitivo e da fragilidade em pessoas idosas, por meio de instrumentos validados como o Mini Exame do Estado Mental e a Escala de Fragilidade de Edmonton, a fim de identificar agravos e intervir precocemente.

\section{REFERÊNCIAS}

1. Miranda GMD, Mendes A da CG, Silva ASLA da. Envelhecimento da população no Brasil: desafios e conseqüências sociais atuais e futuros. Rev.bras. geriatr. gerontol. [Internet]. 2016 [acesso em 04 jun 2018]; 19(3). Disponível em: http://dx.doi.org/10.1590/1809-98232016019.150140.

2. Leonardo KC, Talmelli LFS, Diniz MA, Fhon JRS, Fabrício-Wehbe SCC, Rodrigues RAP. Assessment of cognitive status and frailty of elder elderly living at home. Ciênc. cuid. saúde. [Internet]. 2014 [acesso em 11 maio 2018]; 13(1). Disponível em: http://dx.doi.org/10.4025/cienccuidsaude.v13i1.20033.

3. Rockwood K, Howlett SE, MacKnight C, Beattie BL, Bergman H, Hébert R, et al. Prevalence, attributes and outcomes of fitness and frailty in community-dwelling older adults: report from the Canadian Study of health and aging. J Gerontol A Biol Sci Med sci.med. sci. [Internet]. 2004 [acesso em 11 mai 2018]; 59(12). Disponível em: https://www.ncbi.nlm.nih.gov/pubmed/15699531.

4. Carneiro AJ, Ramos GCF, Barbosa ATF, Mendonça JMG de, Costa FM da, Caldeira AP. Prevalence and factors associated with frailty in non-institutionalized older adults. Rev bras enferm. [Internet]. 2016 [acesso em 15 jun 2018]; 69(3). Disponível em: http://dx.doi.org/10.1590/0034-7167.2016690304i.

5. Zortea B, Gautério-Abreu DP, Santos SSC, Silva BT, Ilha S, Cruz VD. Association between physical frailty and cognitive scores in older adults. Rev RENE. [Internet] 2015 [acesso em 16 maio 2018]; 16(1).

Disponível em: http://dx.doi.org/10.15253/2175-6783.2015000300012.

6. Kang JY, Kim CH, Sung EJ, Shin HC, Shin WJ, Jung KH. The association between frailty and cognition 
in elderly women. Korean J Fam Med. [Internet]. 2016 [acesso em 12 maio 2018]; 37(3). Disponível em: https://doi.org/10.4082/kjfm.2016.37.3.164.

7. Kallenberg MH, Kleinveld HA, Dekker FW, Munster BC, Rabelink TJ, Buren M, et al. Functional and Cognitive Impairment, Frailty, and Adverse Health Outcomes in Older Patients Reaching ESRD-A Systematic Review. Clin J Am Soc Nephrol. [Internet]. 2016 [acesso em 13 maio 2018]; 11(9). Disponível em: https://doi.org/10.2215/CJN.13611215.

8. Mata FAF da, Pereira PP da S, Andrade KRC de, Figueiredo ACMG, Silva MT, Pereira MG. Prevalence of frailty in Latin America and the Caribbean: a systematic review and meta-analysis. PLoS One. [Internet]. 2016 [acesso em 13 maio 2018]; 11(8). Disponível em: https://doi.org/10.1371/journal.pone.0160019.

9. Grden CRB, Barreto MFC, Sousa JAV, Chuertniek JA, Reche PM, Borges PKO. Frailty and cognitive performance of elderly people in outpatient care. Rev Min Enferm. [Internet]. 2018 [acesso em 12 mar 2019]; 22. Disponível em: https://doi.org/10.5935/1415-2762.20180016.

10. Bertolucci PHF, Brucki SMD, Campacci SR, Juliano Y. Mini-exame do estado mental em uma população geral: impacto da escolaridade. Arq. Neuro-Psiquiatr. [Internet]. 1994 [acesso em 19 jun 2018]; 52(1). Disponível em: http://dx.doi.org/10.1590/S0004-282X1994000100001.

11. Fabrício-Wehbe SCC, Schiaveto FV, Vendrusculo TRP, Haas VJ, Dantas RAS, Rodrigues RAP. Crosscultural adaptation and validity of the "edmonton frail scale - efs" in a brazilian elderly sample. Rev Lat Am Enfermagem [Internet]. 2009 [acesso em 12 mai 2018]; 17(6). Disponível em: http://dx.doi. org/10.1590/S0104-11692009000600018.

12. Ministério da Saúde (BR). Conselho Nacional de Saúde. Diretrizes e normas regulamentadoras de pesquisa envolvendo seres humanos. Resolução n. 466, de 12 de dezembro de 2012. Brasília; 2012.

13. Torres JL, Dias RC, Ferreira FR, Macinko J, Lima-Costa MF. Functional performance and social relations among the elderly in Greater Metropolitan Belo Horizonte, Minas Gerais State, Brazil: a population-based epidemiological study. Cad Saude Publica. [Internet]. 2014 [acesso em 12 jun 2018]; 30(5). Disponível em: http://dx.doi.org/10.1590/0102-311X00102013.

14. Lino VTS, Portela MC, Camacho LAB, Rodrigues NCP, Andrade MK de N, O'Dwyer G. Rastreamento de problemas de idosos na atenção primária e proposta de roteiro de triagem com uma abordagem multidimensional. Cad. Saúde Pública. [Internet]. 2016 [acesso em 22 abr 2018]; 32(7). Disponível em: http://dx.doi.org/10.1590/0102-311X00086715.

15. Almeida AV, Mafra SCT, Silva EP da, Kanso S. A Feminização da Velhice: em foco as características socioeconômicas, pessoais e familiares das idosas e o risco social. Textos \& Contextos [Internet]. 2015 [acesso em 22 jun 2018]; 14(1). Disponível em: http://dx.doi.org/10.15448/1677-9509.2015.1.19830.

16. Instituto Brasileiro de Geografia e Estatística (IBGE). Mudança demográfica no Brasil no início do século XXI: subsídios para as projeções da população. [Internet] 2015 [acesso em 14 maio 2018]. Disponível em: https://biblioteca.ibge.gov.br/visualizacao/livros/liv93322.pdf.

17. Sturmer J, Bettinelli LA, Amaral PP, Bortoluzzi EC, Doring Marlene. Sociodemographic and clinical profile of elderly users of family health. J Nurs UFPE on line. [Internet] 2017 [acesso em 13 maio 2018]; 11(Suppl 8). Disponível em: http://dx.doi.org/10.5205/reuol.11135-99435-1-ED.1108sup201707.

18. Bezerra TA, Brito MAA de, Costa KN de FM. Characterization of medication use among elderly people attended at a family health care servisse. Cogitare enferm. [Internet]. 2016 [acesso em 11 jun 2018]; 21(1). Disponível em: http://dx.doi.org/10.5380/ce.v21i1.43011.

19. Martins JB, Lange C, Lemões MAM, Llano PMP de, Santos F dos, Avila JA de. Evaluation of cognitive performance in older adult residents of a rural area. Cogitare enferm. [Internet]. 2016 [acesso em $30 \mathrm{abr}$ 2018]; 21(3). Disponível em: http://dx.doi.org/10.5380/ce.v21i3.48943.

20. Oliveira VT de L, Santos ESS dos, Medeiro NN de A, Ribeiro AA, Pessoa MTG. Perfil Sociodemográfico e Hábitos de Vida de Idosos Portadores de Hipertensão. R bras ci Saúde [Internet]. 2017 [acesso em 13 abr 2018]; 21(1). Disponível em: http://dx.doi.org/10.4034/RBCS.2017.21.01.08. 
21. Eid LP, Leopoldino SAD, Oller GASA de O, Pompeo DA, Martins MA, Gueroni LPB. Fatores relacionados às atividades de autocuidado de pacientes com diabetes mellitus tipo 2. Esc. Anna Nery [Internet]. 2018 [acesso em 13 abr 2018]; 22(4). Disponível em: http://dx.doi.org/10.1590/2177-9465ean-2018-0046.

22. Nunes WA, Dias FA, Nascimento JS, Gomes NC, Tavares DM dos S. Cognition, functionality and depression indicative among elderly. Rev RENE. [Internet]. 2016 [acesso em 11 maio 2018]; 17(1). Disponível em: http://dx.doi.org/10.15253/2175-6783.2016000100014.

23. Rodrigues RAP, Fhon JRS, Huayta VMA, Neira WLF, Pontes MLF, Silva AO, Defina GPC. Frailty syndrome and anthropometric measurements in the elderly living at home. J Aging Res Clin Practice. [Internet]. 2017 [acesso em 11 maio 2018]; 6. Disponível em: http://dx.doi.org/10.14283/jarcp.2017.15.

24. Fhon JRS, Rodrigues RAP, Santos JLF, Diniz MA, Santos EB dos, Almeida VC, et al . Factors associated with frailty in older adults: a longitudinal study. Rev. saúde pública. [Internet]. 2018 [acesso em 11 mar 2019]; 52. Disponível em: http://dx.doi.org/10.11606/s1518-8787.2018052000497.

25. Rodrigues RAP, Fhon JRS, Pontes M de L de F, Silva AO, Haas VJ, Santos JLF. Frailty syndrome among elderly and associated factors: comparison of two cities. Rev. Latino-Am. Enfermagem [Internet]. 2018 [acesso em 11 mar 2019]; 26. Disponível em: http://dx.doi.org/10.1590/1518-8345.2897.3100.

26. Chen S, Honda T, Narazaki K, Chen T, Kishimoto H, Haeuchi Y, et al. Physical frailty is associated with longitudinal decline in global cognitive function in non-demented older adults: a prospective study. J Nutr Health Aging. [Internet] 2018 [acesso em 12 mar 2019]; 22(1). Disponível em: http://dx.doi.org/10.1007/ s12603-017-0924-1.

27. Mari RF, Alves GG, Aerts DRG de C, Camara S. The aging process and health: what middle-aged people think of the issue. Rev Bras Geriatr Gerontol. [Internet] 2016 [acesso em 13 abr 2018]; 19(1). Disponível em: http://dx.doi.org/10.1590/1809-9823.2016.14122.

28. Mello A de C, Engstrom EM, Alves LC. Health-related and socio-demographic factors associated with frailty in the elderly: a systematic literature review. Cad. Saúde Pública. [Internet] 2014 [acesso em 15 jun 2018]; 30(6). Disponível em: http://dx.doi.org/10.1590/0102-311X00148213.

29. St John PD, Tyas SL, Griffith LE, Menec V. The cumulative effect of frailty and cognition on mortality - results of a prospective cohort study. Int Psychogeriatr [Internet]. 2017 [acesso em 13 abr 2018];29(4). Disponível em: http://dx.doi.org/10.1017/S1041610216002088.

Recebido: $18 / 07 / 2018$

Finalizado: 08/08/2019

Autor Correspondente:

Cleane Rosa Ribeiro da Silva

Universidade Federal da Paraíba

Cidade Universitária, s/n - 58051-085, João Pessoa, PB, Brasil

E-mail: cleane_rosas@hotmail.com

Contribuição dos autores:

Contribuições substanciais para a concepção ou desenho do estudo; ou a aquisição, análise ou interpretação de dados do estudo - RRP, CRRS, SCV, LAVB

Elaboração e revisão crítica do conteúdo intelectual do estudo - RRP, CRRS, SCV, EAM

Aprovação da versão final do estudo a ser publicado - LAVB, MLFP

Responsável por todos os aspectos do estudo, assegurando as questões de precisão ou integridade de qualquer parte do estudo - MLFP 